\title{
Total Saponins of Aralia Elata (Miq) Seem Alleviate Calcium Homeostasis Imbalance and Endoplasmic Reticulum Stress- Related Apoptosis Induced by Myocardial Ischemia/Reperfusion Injury
}

\author{
Ruiying Wang ${ }^{a}$ Minghua Yang ${ }^{b}$ Min Wang ${ }^{a}$ Xuesong Liu ${ }^{b}$ Huibo $\mathrm{Xu}^{\mathrm{c}}$ \\ Xudong $\mathrm{Xu}^{\mathrm{a}}$ Guibo Sun ${ }^{\mathrm{a}}$ Xiaobo Sun ${ }^{\mathrm{a}}$ \\ aKey Laboratory of Bioactive Substances and Resources Utilization of Chinese Herbal Medicine, Ministry \\ of Education, Institute of Medicinal Plant Development, Chinese Academy of Medical Sciences \&

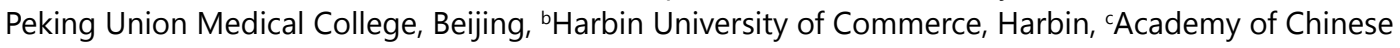 \\ Medical Sciences of Jilin Province, Changchun, China
}

\section{Key Words}

Myocardial ischemia/reperfusion • Calcium homeostasis $•$ Endoplasmic reticulum stress $\bullet$ Total saponins

\begin{abstract}
Background/Aims: Total saponins of Aralia elata (Miq) Seem (AS) from the Chinese traditional herb Long ya Aralia chinensis L. reportedly provide cardioprotective effects, but the exact mechanisms require further study. Previous studies have showed that myocardial ischemia/ reperfusion injury (MIRI) was related to calcium homeostasis imbalance and endoplasmic reticulum stress (ERS). Thus, this study aimed to demonstrate protective effects of AS on MIRI. Methods: After administrating AS for 5 days, the left anterior descending artery coronary artery of Sprague-Dawley (SD) rats was ligated for $30 \mathrm{~min}$. After $48 \mathrm{~h}$ of reperfusion, haemodynamics, Evans blue/ 2,3,5-triphenyltetrazolium chloride (TTC) staining, hematoxylineosin (HE) staining, masson staining and the levels of lactate dehydrogenase (LDH) and creatine kinase (CK), superoxide dismutase (SOD), malondialdehyde (MDA) were detected to assess MIRI. ATPase activity and Western Blot were used to study the mechanisms. Results: Compared with IR group, AS treatment groups could significantly reduce myocardial infarct size; improve myocardial pathologic progress; decrease content of $\mathrm{LDH}, \mathrm{CK}$, and MDA; increase content of SOD; and restore the activities of $\mathrm{Ca}^{2+}-\mathrm{Mg}^{2+}$-ATPase, $\mathrm{Na}^{+}-\mathrm{K}^{+}$-ATPase, sarcoplasmic reticulum $\mathrm{Ca}^{2+}$-ATPases (SERCA), and calcineurin $(\mathrm{CaN})$. AS treatment groups also significantly up-regulated the expression of GRP78, C/EBP homologous protein (CHOP), and Bax, and

R. Wang and M. Yang contributed equally to this work.

\begin{tabular}{ll}
\hline Xudong Xu, Guibo Sun & Institute of Medicinal Plant Development (IMPLAD), Chinese Academy of Medical Sciences \& Peking \\
and Xiaobo Sun & Union Medical Colleg, No. 151, Malianwa North Road, Haidian District, Beijing, 100193 (China) \\
& Fax+8610 57833013, E-Mail xdxu@implad.ac.cn; sunguibo@126.com; sun_xiaobo163@163.com
\end{tabular}
\end{abstract}


down-regulated the expression of $\mathrm{BCl}-2$, all similar to the effects of ERS. Conclusion: These findings illustrated that AS could prevent myocardial ischemia/reperfusion injury and reduce calcium homeostasis imbalance and ERS-related apoptosis.

(C) 2018 The Author(s)

Published by S. Karger AG, Basel

\section{Introduction}

Ischemic heart disease is a major cause of morbidity and mortality worldwide [1, 2]. Clinical study has shown that sometimes reperfusion after ischemia can't improve the condition, and instead exacerbates the damage, which is known as myocardial ischemia/ reperfusion injury (MIRI) [3]. MIRI induces myocardial cell apoptosis and necrosis, and increased infarct size, which can lead to arrhythmias, heart failure and other cardiovascular disease, and reduce the probability of cure after thrombolytic therapy [4]. Therefore, achieving both early recovery of ischemic tissue flow and no or lesser reperfusion injury in ischemic disease are crucial. During myocardial ischemia/reperfusion, the shortage of oxygen and energy breaks the homeostasis of the endoplasmic reticulum known as endoplasmic reticulum stress (ERS), which causes unfolded protein response (UPR)[4, 5]. Along with the prolonged and severe ER stress, apoptotic signaling will be activated. Moreover, under reperfusion injury, abundant reactive oxygen species (ROS) cause cell membrane damage and its permeability increasing, leading to extracellular $\mathrm{Ca}^{2+}$ influx. Eventually, the injury results in intracellular calcium overload, which causes myocardial contraction disorders, apoptosis and other damage reactions [6]. It's obvious that calcium homeostasis and ERS play a role in the mechanisms of MIRI.

Aralia elata (Miq) Seem is widely distributed in the Asian region, especially in the northeast China [7]. It is an important folk medicine for the treatment of diabetes, arthritis, myocardium infarction and other diseases. The total saponins of $A$. elata (aralosides, AS) are the main active components of A. elata (Miq) Seem [7, 8]. Specially, Longya Guanxinkang capsule, whose main component was AS, was proven to have the function of supplementing qi to activate blood and removing blood stasis to relieve pain, and be applied to treat the syndrome of blood stasis due to qi deficiency [2]. In addition, our group accomplished some research on AS, and demonstrated the characterization of AS using mass spectrometry and the positive inotropic effect of AS on canine myocardium and isolated rat cardiomyocytes, suggesting that AS can regulate $\mathrm{Ca}^{2+}$ in myocardial cell [9]. The study of our group also showed negative inflammatory responses and apoptotic activity of AS on liver tissue of ApoE-/-mice [10], and revealed that AS was associated with apoptosis and ERS. However, the involvement of the protective effects of AS in calcium homeostasis and ERS remains unclear.

In this study, we investigated whether AS protects against MIRI by regulating calcium homeostasis and ERS-related apoptosis pathway. A MIRI model involving $30 \mathrm{~min}$ ischemia and $48 \mathrm{~h}$ reperfusion was established.

\section{Materials and Methods}

Plant material, extraction and isolation of AS

The roots of AS were collected from Jilin Province of China in September 2010. The samples were identified by Professor Zhong Kai Yan (Academy of Chinese Medical Sciences of Jilin Province). A voucher specimen (No. 20100920) was deposited in the same department. The isolation methods of AS were coincided with the previous study $[10,11]$.

UPLC analysis and mass spectrometry

The total saponins were separated on an Acquity UPLCTM system (Waters Corp., USA), and measured by Mass spectrometry on a Synapt G2 MS system (Waters Corp., USA) equipped with an ESI source as previously reported $[10,11]$. 


\section{Cellular Physiology Cell Physiol Biochem 2018;50:28-40 and Biochemistry Published \begin{tabular}{l|l} 
DOI: 10.1159/000493954 & $\begin{array}{l}\text { (c) } 2018 \text { The Author(s). Published by S. Karger AG, Basel } \\
\text { www.karger.com/cpb }\end{array}$
\end{tabular}}

Wang et al.: AS Protects MIRI from Calcium Homeostasis Imbalance and ERS

Animals

Adult male Sprague-Dawley (SD) rats (body weight 260-300g) were obtained from Beijing Vital River Laboratory Animal Technology Co., Ltd., Beijing, China. The animals were provided free access to food and water, under standard laboratory conditions (temperature: $22^{\circ} \mathrm{C} \pm 1{ }^{\circ} \mathrm{C}$; humidity: $60 \%$ ) with a $12 \mathrm{~h}$ light/12h dark cycle in conventional cages. All the procedures were approved by the Laboratory Animal Ethics Committee of the Institute of Medicinal Plant Development, Peking Union Medical College. Procedures and interventions conform to NIH Guidelines for the Care and Use of Laboratory Animals.

\section{Experimental groups}

Longya Guanxinkang capsule' s clinical dose $540 \mathrm{mg} / \mathrm{kg}$ also provided the basis for the experimental dose determination. After pretests, rat doses above were finally determined as $25,50,100 \mathrm{mg} / \mathrm{kg} / \mathrm{day}$. Therefore, animal experimental groups were as followed: (1) sham group; (2) ischemia/reperfusion(I/R) group; (3) AS 100mg/kg/day group; (4) AS 100mg/kg/day +I/R group; (5) AS 50mg/kg/day +I/R group; (6) AS 25mg/kg/day +I/R group; (7) Captopril 20mg/kg/day +I/R. AS and Captopril were prepared using ultrapure water. All the rats were orally administered by gavage for 5 days before surgery, and Sham and I/R groups were administered with the same amount of ultrapure water for 5 days.

\section{Experimental model of myocardial I/R injury}

Rats were anesthetized with an intraperitoneal injection of pentobarbital sodium (40mg/kg) and ventilated using a small animal ventilator at the rate of 70 breaths per minute. The left anterior descending (LAD) coronary artery was ligated with a 6-0 silk suture over a polyethylene tube for $30 \mathrm{~min}$ [12]. Unfastening of the suture signified reperfusion lasting $48 \mathrm{~h}$. The same surgical procedures were subjected to sham group and AS $100 \mathrm{mg} / \mathrm{kg} /$ day group, except for not fastening the suture around the LAD.

\section{Assessment of myocardial infarct size}

At the end of reperfusion, the LAD was ligated in the same position again and $2 \mathrm{~mL}$ of $4 \%$ Evans blue dye was injected from the postcava. Then the heart was immediately taken out and placed in the freezer at $-80^{\circ} \mathrm{C}$ for $7 \mathrm{~min}$. The frozen heart was cut into $2-3 \mathrm{~mm}$-thick slices along the direction of the ligature. The slices were stained in 2, 3,5-triphenyl-2H-tetrazolium chloride (TTC) solution (1\%) for 12 min at $37^{\circ} \mathrm{C}$, and then fixed in $4 \%$ paraformaldehyde overnight [13]. The infarct area was calculated with ImageJ software after images were taken with a high-definition camera.

\section{Measurement of hematoxylin-eosin (HE) staining}

After $48 \mathrm{~h}$ reperfusion, the hearts were rapidly removed and rinsed, and fixed in $4 \%$ paraformaldehyde for $48 \mathrm{~h}$ [14]. Then they were dehydrated in graded ethanol and embedded in paraffin max, then sliced and stained with hematoxylin and eosin. These slices were observed under a microscope with $\times 400$ magnification.

\section{Measurement of masson staining}

After $48 \mathrm{~h}$ reperfusion, the hearts were perfused with $4 \%$ paraformaldehyde to remove the residual blood, and fixed in $4 \%$ paraformaldehyde for $48 \mathrm{~h}$ before embedding in paraffin [14]. All slices were cross sections of the heart and stained with hematoxylin, aniline blue and acid magenta. These slices were observed under a microscope with $\times 400$ magnification. The fibrotic areas were calculated with HistoQuest tissue analysis software.

\section{Determination of $L D H$ and $C K$}

After $48 \mathrm{~h}$ reperfusion, blood samples were collected and centrifuged at $3000 \mathrm{rpm}$ for $15 \mathrm{~min}$. The upper serum was left for the detection of lactate dehydrogenase (LDH) and creatine kinase (CK) using assay kits (Jiancheng Biological Engineering Institute, Nanjing, China) [14]. Detailed manipulation processes were performed according to the manufacturer's instructions. All samples were tested in duplicate.

\section{Determination of MDA and SOD}

After $48 \mathrm{~h}$ reperfusion, heart tissues were collected and homogenized $(10 \% \mathrm{w} / \mathrm{v})$ with physiological saline and centrifuged at $3000 \mathrm{rpm}$ for $15 \mathrm{~min}$ [7]. The supernatant was used to determination of determine malondialdehyde (MDA) and superoxide dismutase (SOD) in heart tissue using assay kits (Jiancheng 


\section{Cellular Physiology Cell Physiol Biochem 2018;50:28-40 and Biochemistry Published onIne: 3 October 2018 \begin{tabular}{l|l} 
DOI: 10.1159/000493954 & $\begin{array}{l}\text { C } 2018 \text { The Author(s). Published by S. Karger AG, Basel } \\
\text { www.karger.com/cpb }\end{array}$
\end{tabular}}

Wang et al.: AS Protects MIRI from Calcium Homeostasis Imbalance and ERS

Biological Engineering Institute, Nanjing, China). Detailed manipulation processes were performed according to the manufacturer's instructions. All samples were tested in duplicate.

\section{Measurement of haemodynamics}

At the end of reperfusion, rats were anesthetized, then connected to the small animal ventilator and electrocardiograph. The biosensor was inserted into the left ventricle from the right common carotid artery [15]. The hemodynamic data of the rats were recorded: left ventricular end diastolic pressure (LVEDP), left ventricular systolic pressure (LVSP), $\max \mathrm{dP} / \mathrm{dt}$, $\min \mathrm{dP} / \mathrm{dt}$, and heart rate.

Activity assay of $\mathrm{Ca}^{2+}-\mathrm{Mg}^{2+}$-ATPase, $\mathrm{Na}^{+}-\mathrm{K}^{+}$-ATPase, sarcoplasmic reticulum $\mathrm{Ca}^{2+}$-ATPases and Calcineurin in heart tissue

After reperfusion, the heart tissues below the ligature were used for the following experiment. The tissues were added 9 times normal saline in a ratio of 1:9 by weight, mechanically homogenized in an icewater bath, and centrifuged at $2500 \mathrm{rpm}$ for $10 \mathrm{~min}$. The supernatant was used to detect the activities of $\mathrm{Ca}^{2+}-\mathrm{Mg}^{2+}$-ATPase, $\mathrm{Na}^{+}-\mathrm{K}^{+}$-ATPase, sarcoplasmic reticulum $\mathrm{Ca}^{2+}$-ATPases (SERCA) and calcineurin (CaN) using assay kits [16] (Jiancheng Biological Engineering Institute, Nanjing, China). Detailed manipulation processes were performed according to the manufacturer's instructions. All samples were operated in duplicate.

Western blot analysis

After $48 \mathrm{~h}$ reperfusion, total protein was extracted from the myocardial infarction area using mammalian protein extraction kits (Kangwei Century Biotechnology Institute, Beijing, China), and protein concentration was determined using a BCA kit (Kangwei Century Biotechnology Institute, Beijing, China). Equal protein samples of all the groups were separated using SDS-PAGE and transferred onto nitrocellulose membranes. The membranes were incubation with primary antibodies overnight at $4{ }^{\circ} \mathrm{C}$, and then incubated with corresponding secondary antibodies for $2 \mathrm{~h}$ at room temperature $[10,11]$. Immunoblots were developed using an ECL kit (Kangwei Century Biotechnology Institute, Beijing, China). The intensities of band were analyzed using the Gel Pro software (Media Cybernetics, Rockville, MD, USA). The main antibodies used in the experiments were obtained from abcam and catalogue numbers were as follows: GRP78 (ab108615), CHOP (ab11419), Bax (ab32503), Bcl-2 (ab32124), GAPDH (ab8245).

\section{Statistical analysis}

All experiments were repeated at least three times. Data were expressed as mean \pm SD and analyzed by one-way ANOVA followed by Newman-Keuls multiple comparison test as appropriate (GraphPad Prism version 5 software). $\mathrm{P}<0.05$ was considered statistically significant.

\section{Results}

Characterization of the saponins in the active fraction

UPLC/Q-TOF-MS analysis showed that the active fraction contained high amounts of saponins as previously reported [11]. Approximately 30 visible peaks could be determined from the total ion current profile of the active fraction; among these peaks, 16 were identified. The compounds were characterized in terms of retention times and mass spectra, and then were identified by comparing with published data or commercial standards. UPLC result of AS sample is shown in Fig. 1. A complete list summarizing all of the compounds identified in the active fraction is shown in Table 1. Therefore, 16 compounds identified could be considered as the main components of the efficacy; however, those compounds that have not been identified may still play an important role in the protection of cardiomyocytes.

\section{Effect of AS on infarct size}

To investigate the effects of AS on MIRI in SD rats, we dyed the hearts of rats with Evans blue for myocardial area at risk(blue) and TTC for myocardial infarct area(white) after the reperfusion [13]. After five days' administration of AS, especially high concentration, the infarct size became smaller than the I/R group, whereas the other concentrations had no 
significant difference, as shown in Fig. 2. The results showed that AS reduced infarct size after myocardial IR.

Effect of AS on HE and masson staining

In the $\mathrm{HE}$ staining assay, the sham and AS group were shown no difference compared with normal tissue without characteristic lesions. After IR, the hearts exhibited hyperemia, hemorrhage, reparative inflammation, myocardial fibrosis, and even necrosis in Table 2 . After treatment with AS $(25,50,100 \mathrm{mg} / \mathrm{kg} /$ day $)$, pathological changes could be reduced to a certain extent, but the lesion was inevitable in Fig. 3, which showed that AS could protect the myocardium from IR injure [17]. In masson staining, the normal cardiac myocytes were stained red and fibrotic areas were stained blue. The blue areas were almost none in sham and AS $100 \mathrm{mg} / \mathrm{kg} /$ day group, whereas increased in the other groups showing obvious fibrosis in Fig. 4 (A). Furthermore, fibrotic areas in $\mathrm{AS}+\mathrm{I} / \mathrm{R}$ groups

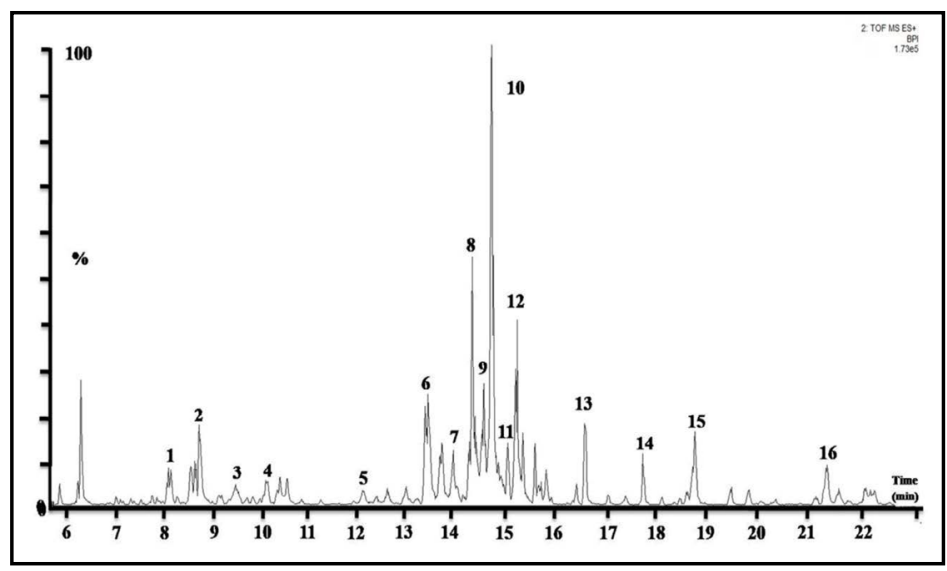

Fig. 1. Total ion current profile corresponding to the UPLC-ESI-MS analysis of the total saponins of Aralia elata (AS).

Table 1. List of saponins identified in the active fraction of Aralia elata

\begin{tabular}{|c|c|c|c|}
\hline \#Peak & Chemical name & {$[\mathrm{M}+\mathrm{Na}]^{+}$} & Fragments and their intensity (\%) \\
\hline 1 & Elatoside L & 1435.6212 & $\begin{array}{c}1303.5857(6.2), 1141.5377(10.2), 965.5003(18.2), \\
949.4755(12.6), 729.3074(8.6), 637.2259(5.4), \\
509.1479(7.4), 439.3572(14.8)\end{array}$ \\
\hline 2 & Congmunsoides $\mathrm{X}$ & 1303.5846 & $\begin{array}{l}1141.5305(12.2), 965.5045(9.3), 817.4280(8.6) \\
649.2185(3.2), 509.1446(10.6), 439.3559(46.8)\end{array}$ \\
\hline 3 & Congmunsoides V & 1127.5171 & $\begin{array}{c}965.4675(5.2), 863.3168(7.6), 537.2616(16.3) \\
515.2830(62.4), 493.1171(37.8), 443.2600(100)\end{array}$ \\
\hline 4 & Congmuosides XI & 1273.5757 & $\begin{array}{l}1141.5369(32.5), 979.4843(8.8), 949.4748(8.6) \\
803.4537(33.2), 493.1159(4.6), 439.3557 \text { (42.8) }\end{array}$ \\
\hline 5 & Congmunsoides G & 1317.6060 & $\begin{array}{c}1109.5093(54.2), 807.4851(100), 721.4186(26.8), \\
670.2960(35.4), 493.1150(43.2), 369.1950(52.0)\end{array}$ \\
\hline 6 & Elatoside D & 1141.5367 & $\begin{array}{c}979.4832(22.6), 795.4523(5.6), 557.1013(4.2) \\
523.1266(45.4), 439.3564(20.3)\end{array}$ \\
\hline 7 & Tarasaponin IV & 1111.5275 & $\begin{array}{c}949.4787(6.6), 949.4739(10.4), 641.4011(5.2) \\
493.1174(16.2), 439.3564(26.2)\end{array}$ \\
\hline 8 & Elatoside C & 1111.5276 & $\begin{array}{c}949.4750(16.8), 641.4030(8.2), 493.1162(46.2) \\
439.3575(6.8)\end{array}$ \\
\hline 9 & Elatoside $\mathrm{K}$ & 1111.5264 & $\begin{array}{c}949.4754(26.2), 641.4035(5.2), 493.1164(48.5) \\
439.3566(26.8)\end{array}$ \\
\hline 10 & Aralosdie C & 1111.5283 & $\begin{array}{c}979.4855(11.9), 949.4731(26.8), 641.4010(18.5) \\
493.1164(36.4), 439.3566(14.2)\end{array}$ \\
\hline 11 & Elatoside I & 979.4857 & $\begin{array}{c}817.4307(16.3), 641.4307(8.4), 439.3553(22.6) \\
361.0741(21.4)\end{array}$ \\
\hline 12 & Spinasaponin A 28-O-Glc & 979.4856 & $\begin{array}{c}817.4324(16.3), 817.4307(17.6), 641.4022(18.8) \\
439.3566(32.6), 361.0745(31.4)\end{array}$ \\
\hline 13 & Araloside A & 949.4743 & 787.4208 (11.5), 641.4016 (22.3), 439.3569 (32.6) \\
\hline 14 & Ginsenoside Rd & 969.5341 & 789.4738 (33.5), 425.3737 (6.8), 407.3669 (12.3) \\
\hline 15 & Chikusetsusapnoin IV & 965.5048 & $\begin{array}{c}831.4465 \text { (2.8), } 509.1452(14.5), 491.2415 \text { (15.6), } \\
439.3557(26.2)\end{array}$ \\
\hline 16 & Chikusetsusaponin IV & 949.4813 & 795.4448 (22.7), $493.1172(34.9), 439.3530$ (21.6) \\
\hline
\end{tabular}
Captopril group in Fig. 4 (B) [18]. The results of masson staining were consistent with HE staining.

\section{Effect of AS on $L D H, C K, M D A$ and SOD}

During myocardial infarction, $\mathrm{LDH}$ and CK will leak from myocardial tissues to the blood [19]. As shown in Fig. 5 (A, B), the I/R group significantly increased the level of LDH and CK in the serum of rats compared with the sham group, but pretreatment with different doses of AS could markedly downregulate the levels of CK and LDH in different degrees. Lipid peroxidation and oxidative damage by free radicals in IR injury could be assessed by the level of MDA. SOD is an important antioxidant enzyme in the body, which can indirectly reflect the degree of cell oxidation damage. The results presented in Fig. 5 (C, D) indicated that IR caused significant increases in MDA activities and decrease in SOD compared with the sham group. However, treatment of AS in different doses could depress the changes induced by 


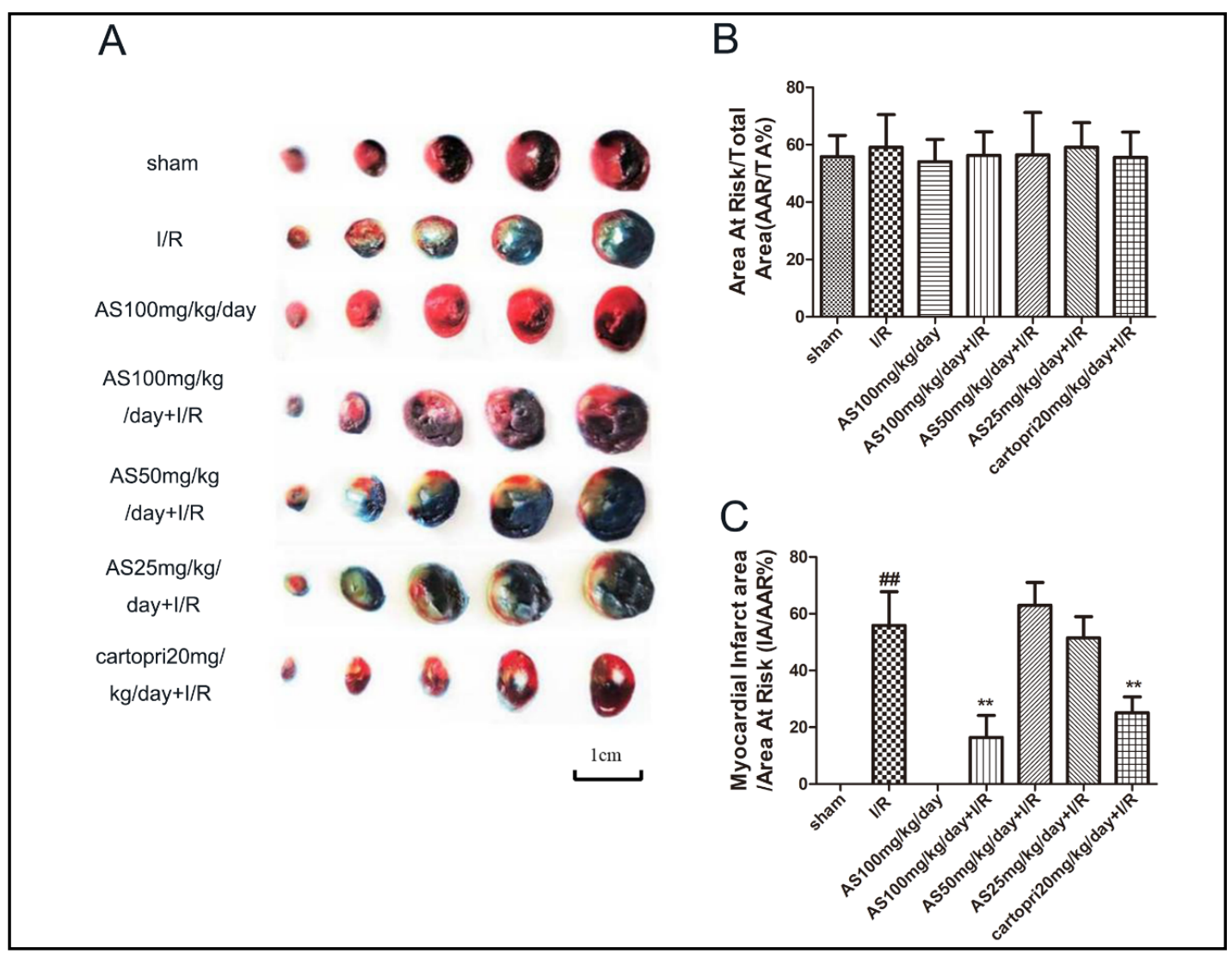

Fig. 2. The total saponins of Aralia elata (AS) reduced infarct size after myocardial I/R. (A) Representative photographs of Evans blue/TTC double stained heart slices obtained $48 \mathrm{~h}$ after MIRI. Scale bar is $1 \mathrm{~cm}$. (BC) Graphic representation of the infarct size. All data expressed as mean $\pm S D, n=6 /$ group, ${ }^{\# \#} \mathrm{P}<0.01$ vs. sham group; ${ }^{* *} \mathrm{P}<0.01$ vs. I/R group. Abbreviation: AAR, area at risk(blue); IA, infarct area(white).

MIRI. Taken together, these results suggested that AS could alleviate IR-induced oxidative damage.

\section{Effect of AS on} haemodynamics

To understand the protective role of AS on MIRI, we performed hemodynamics to detect indices including LVEDP, LVSP, $+\mathrm{dP} / \mathrm{dt}$ and $-\mathrm{dP} / \mathrm{dt}$ [20]. As illustrated in Fig. 6 , and comparing with the sham group, we found that three indices LVSP, $+\mathrm{dp} / \mathrm{dt}$, $-\mathrm{dp} / \mathrm{dt}$ in the I/R group were significantly reduced, but LVEDP was elevated. The AS+I/R group exhibited a significantly dose-dependent decrease in LVEDP, while no significant changes were found in LVSP, $+\mathrm{dp} / \mathrm{dt},-\mathrm{dp} / \mathrm{dt}$ compared with the IR group.
Table 2. The results of Pathological indicators in HE staining. Attachment: The scoring criteria was as follows: 0: no lesion;m0-1: lesions were less than 1/4 of the designated area; 1-2: lesions ranged from approximately $1 / 4$ to $1 / 2$ of the designated area; $2-3$ : lesions ranged from approximately $1 / 2$ to $3 / 4$ of the designated area; $3-4$ : lesions were greater than $3 / 4$ of the designated area. All data expressed as mean $\pm \mathrm{SD}, \mathrm{n}=3$ /group, ${ }^{\# \#} \mathrm{P}<0.001$, \#\# $\mathrm{P}<0.01$ vs. sham group; ${ }^{*} \mathrm{P}<0.1$, ${ }^{* *} \mathrm{P}<0.01$ vs. I/R group

\begin{tabular}{lccccc}
\hline & hyperemia & hemorrhage & fibrosis & necrosis & degeneration \\
\hline Sham & $1 \pm 0$ & $0 \pm 0$ & $0 \pm 0$ & $0 \pm 0$ & $1 \pm 0$ \\
I/R & $3.67 \pm 0.58^{\# \#}$ & $0.67 \pm 0.58$ & $3.33 \pm 0.58^{\# \# \#}$ & $4 \pm 0^{\# \# \#}$ & $4 \pm 0^{\# \# \#}$ \\
As $100 \mathrm{mg} / \mathrm{kg}$ & $1 \pm 0$ & $0 \pm 0$ & $0 \pm 0$ & $0 \pm 0$ & $1 \pm 0$ \\
As100mg/kg+l/R & $2.67 \pm 0.58$ & $0 \pm 0$ & $2 \pm 1^{*}$ & $2.67 \pm 0.33^{* *}$ & $2.67 \pm 0.33^{* *}$ \\
As50mg/kg+I/R & $2.33 \pm 1.16$ & $0 \pm 0$ & $3.67 \pm 0.57$ & $3 \pm 0$ & $3 \pm 0$ \\
As25mg/kg+I/R & $2.33 \pm 0.58$ & $0 \pm 0$ & $2.33 \pm 0.57$ & $2.67 \pm 0.33^{* *}$ & $2.67 \pm 0.33^{* *}$ \\
Cartopri20mg/kg+I/R & $3 \pm 1$ & $0.33 \pm 0.58$ & $3.67 \pm 0.57$ & $3.67 \pm 0.33$ & $3.67 \pm 0.33$ \\
\hline
\end{tabular}




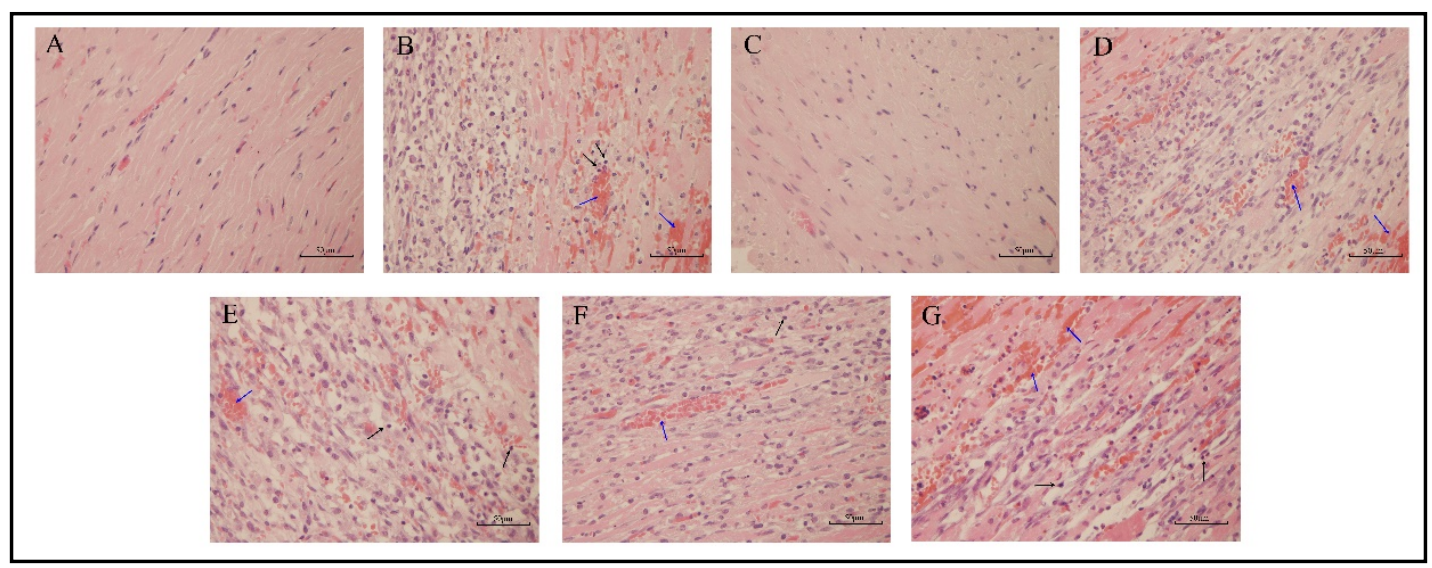

Fig. 3. HE staining showed cardioprotection of the total saponins of Aralia elata (AS) in the IR-impaired hearts(400x). Scale bar is $50 \mu \mathrm{m}$. (A) Sham group; (B) IR group; (C) AS 100 mg/kg/day group; (D) IR+As $100 \mathrm{mg} / \mathrm{kg} /$ day group; (E) IR+As $50 \mathrm{mg} / \mathrm{kg} /$ day group; (F) IR+As $25 \mathrm{mg} / \mathrm{kg} /$ day group; (G) IR+ Captopril $20 \mathrm{mg} / \mathrm{kg} /$ day group. Black arrows indicated inflammatory cell infiltration, and blue arrows did hyperemia.

Fig. 4. Masson staining showed cardioprotection of the total saponins of Aralia elata (AS) in the IRimpaired hearts $(400 \times)$. Scale bar is $50 \mu \mathrm{m}$. (A) Representation of each group as following: a. Sham group; b. IR group; c. AS $100 \mathrm{mg} / \mathrm{kg} /$ day group; d. As 100mg/ $\mathrm{kg} /$ day + I/R group; e. As $50 \mathrm{mg} / \mathrm{kg} /$ day + I/R group; f. As 25mg/ $\mathrm{kg} /$ day + I/R group; g. IR+ Captopril 20mg/ $\mathrm{kg} /$ day $+\mathrm{I} / \mathrm{R}$ group. (B) Quantification of fibrosis areas. All data expressed as mean $\pm S D, n=6 /$ group, $\# \# \mathrm{P}<0.001$ vs. sham group; ${ }^{* *} \mathrm{P}<0.001,{ }^{*} \mathrm{P}<0.1$

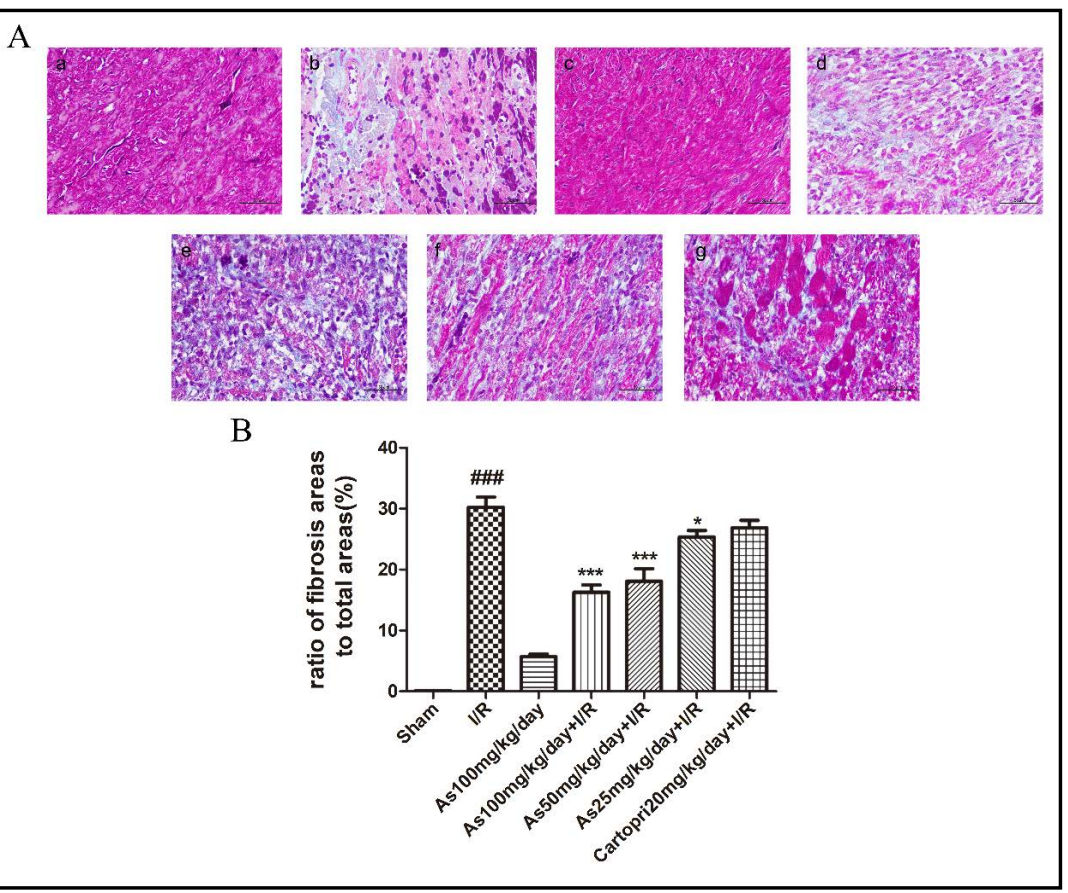
vs. I/R group.

\section{Effect of AS on $\mathrm{Ca}^{2+}-\mathrm{Mg}^{2+}$-ATPase, $\mathrm{Na}^{+}-\mathrm{K}^{+}$-ATPase, SERCA and CaN}

Some studies showed $\mathrm{Na}^{+}-\mathrm{K}^{+}$-ATPase and $\mathrm{Ca}^{2+}-\mathrm{Mg}^{2+}$-ATPase activities were related to $\mathrm{Ca}^{2+}$ input [21]; myocardial $\mathrm{Na}^{+}-\mathrm{K}^{+}$-ATPase and $\mathrm{Ca}^{2+}-\mathrm{Mg}^{2+}$-ATPase activities in the I/R group were markedly lower than those in sham group, as shown in Fig. 7 (A, B). After treatment of AS, the activities significantly recovered. CaN could be activated by calcium-calmodulin, and it was essential for a number of signal transduction pathways [22]. The results in Fig. 7 (C) demonstrated that CaN activity of I/R group increased notably, in contrast with other groups. Given that inhibition of SERCA activity could induce ER stress, MIRI caused by ER stress can be attenuated by preservation of SERCA activity [23]. As shown in Fig. 7 (D), SERCA activity decreased in the I/R group compared with the sham group, whereas it increased in the AS+I/R groups compared with the I/R group. 
Fig. 5. Effect of the total saponins of Aralia elata (AS) on myocardial enzyme activities and lipid peroxidation. (A) The effect of AS on CK level; (B) the effect of AS on LDH level; (C)the effect of AS on MDA level; (D) the effect of AS on SOD level. All data expressed as mean $\pm S D, n=6$ / group, ${ }^{\# \# \# ~} \mathrm{P}<0.001,{ }^{\#} \mathrm{P}<0.05$ vs. sham group; ${ }^{* *} \mathrm{P}<0.001,{ }^{* *} \mathrm{P}<0.01$, ${ }^{*} \mathrm{P}<0.05$ vs. I/R group.

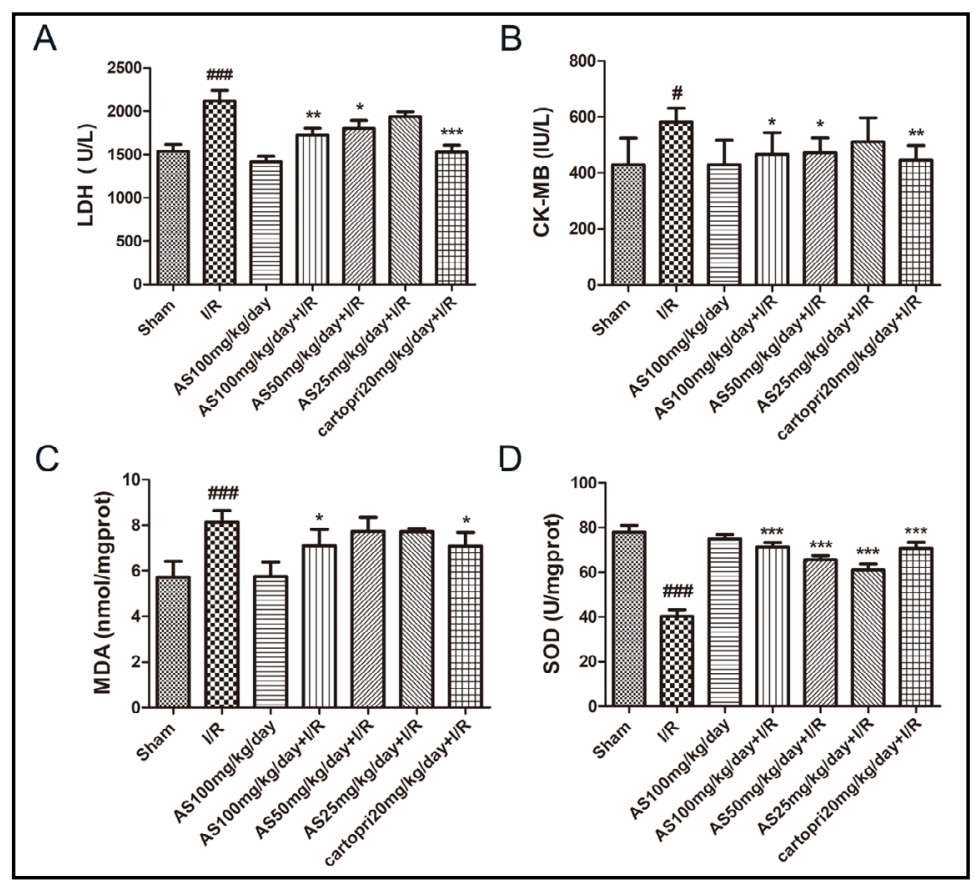

Fig. 6. Effect of the total saponins of Aralia elata (AS) on haemodynamics. (A) LVSP, the left ventricular systolic pressure; (B) LVEDP, the left ventricular end-diastolic pressure; $(C)+d p /$ $\mathrm{dt}$, the maximal rates of pressure rise; (D) $-\mathrm{dp} / \mathrm{dt}$, the maximal rates of pressure fall. All data expressed as mean $\pm S D, n=6 /$ group, "\#\# $\mathrm{P}<0.001$, \#\# $\mathrm{P}<0.01$ vs. sham group; ${ }^{* * *} \mathrm{P}<0.001,{ }^{* *} \mathrm{P}<0.01$, ${ }^{*} \mathrm{P}<0.05$ vs. I/R group.

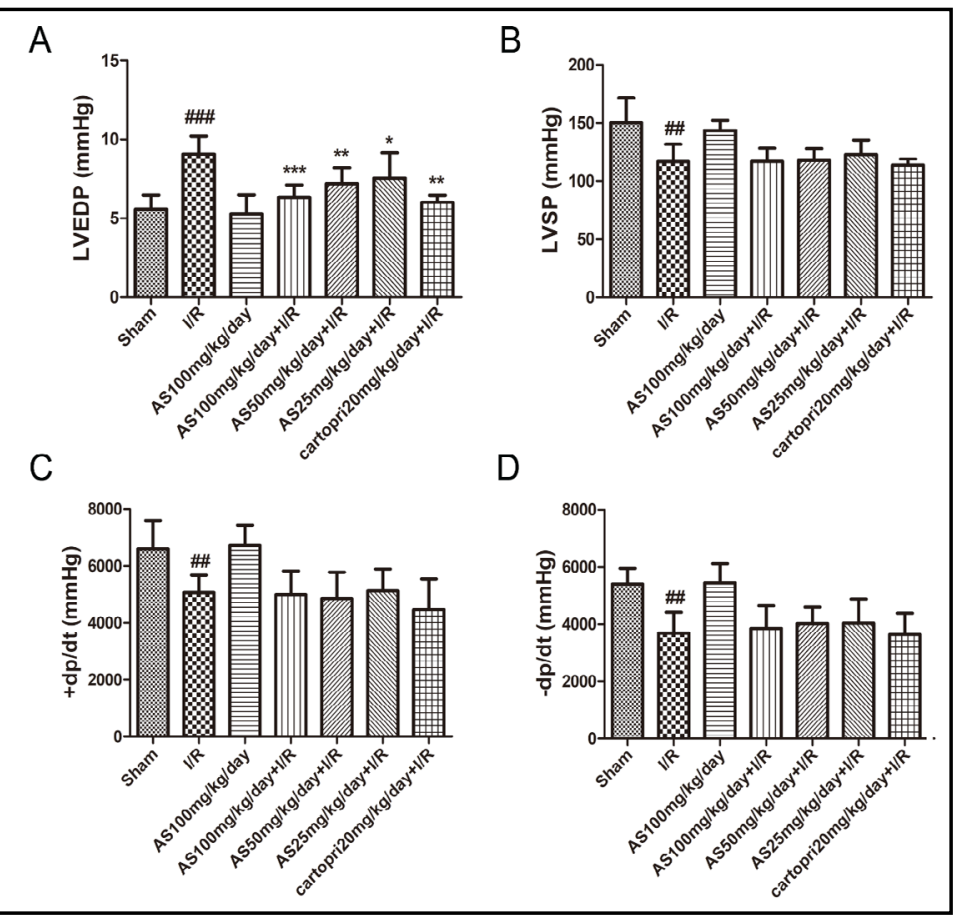

\section{Effect of AS on the expression of proteins related to ERS}

ERS played an important role in MIRI; therefore, we further investigated if AS could reduce MIRI by ERS and associated apoptosis. As shown in Fig. 8, the rat myocardial protein levels of the glucose-regulated protein 78 (GRP78), C/EBP homologous protein (CHOP) and Bax were significantly upregulated in the I/R group compared with the sham group, while Bcl-2 was downregulated. In contrast with the I/R group, as $100 \mathrm{mg} / \mathrm{kg} /$ day $+\mathrm{I} / \mathrm{R}$ group displayed significantly variation in the protein levels of GRP78, CHOP, Bcl-2 and Bax [19]. 
Fig. 7. Effect of the total saponins of Aralia elata (AS) on (A) $\mathrm{Na}^{+}-\mathrm{K}^{+}$-ATPase, (B) $\mathrm{Ca}^{2+}-\mathrm{Mg}^{2+}-$ ATPase, (C) Calcineurin(CaN) and (D) sarcoplasmic reticulum $\mathrm{Ca}^{2+}$-ATPases(SERCA). All data expressed as mean $\pm \mathrm{SD}, \mathrm{n}=6 /$ group, ${ }^{\# \#} \mathrm{P}<0.001$, ${ }^{\# \#} \mathrm{P}<0.01$ vs. sham group; ${ }^{* * *} \mathrm{P}<0.001,{ }^{* *} \mathrm{P}<0.01$ vs. I/R group.

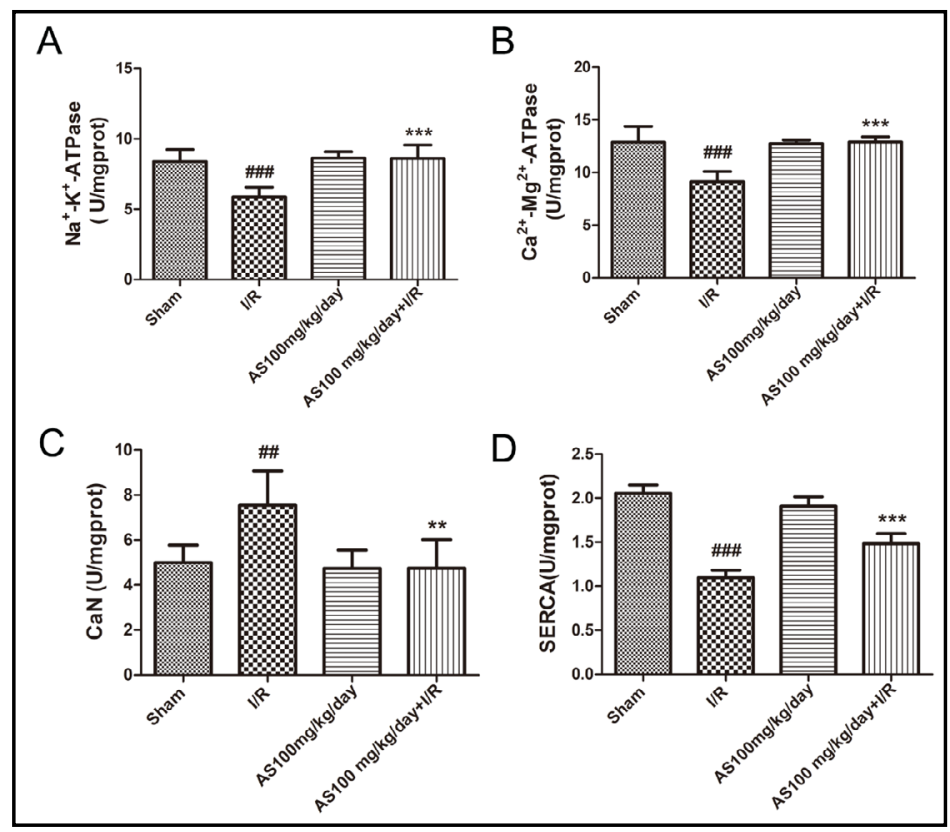

Fig. 8. Effect of Aralia elata (AS) on expression of ERS-associated apoptosis proteins.(A) Myocardial Bcl-2, Bax, chop and GRP78 expression was assayed by western blot analysis. (B)Quantification of protein expression. All data expressed as mean $\pm \mathrm{SD}, \mathrm{n}=3$ /group, ${ }^{\# \# \# ~} \mathrm{P}<0.001$ , ${ }^{\# \# P}<0.01$ vs. sham group; ${ }^{* * *} \mathrm{P}<0.001$, ${ }^{* *} \mathrm{P}<0.01$ vs. I/R group.

\section{Discussion}

AS is main active ingredient of Aralia elata (Miq) Seem, exhibiting anti-aging, antimyocardial ischemia, liverprotection, anti-cancer, antivirus, Anti-inflammation effects [9]. Our previous studies proved that AS had obvious protective effects on acute myocardial ischemia/reperfusion on primary rat cardiomyocytes, which was related to reduce myocardial work and oxygen consumption, increase antioxidant enzyme activitives of myocardial cells, and scavenge free radical $[8,9]$. In addition, our team previously detected myocardial systolic/ diastolic function and calcium transient function and concluded that AS protected against MIRI

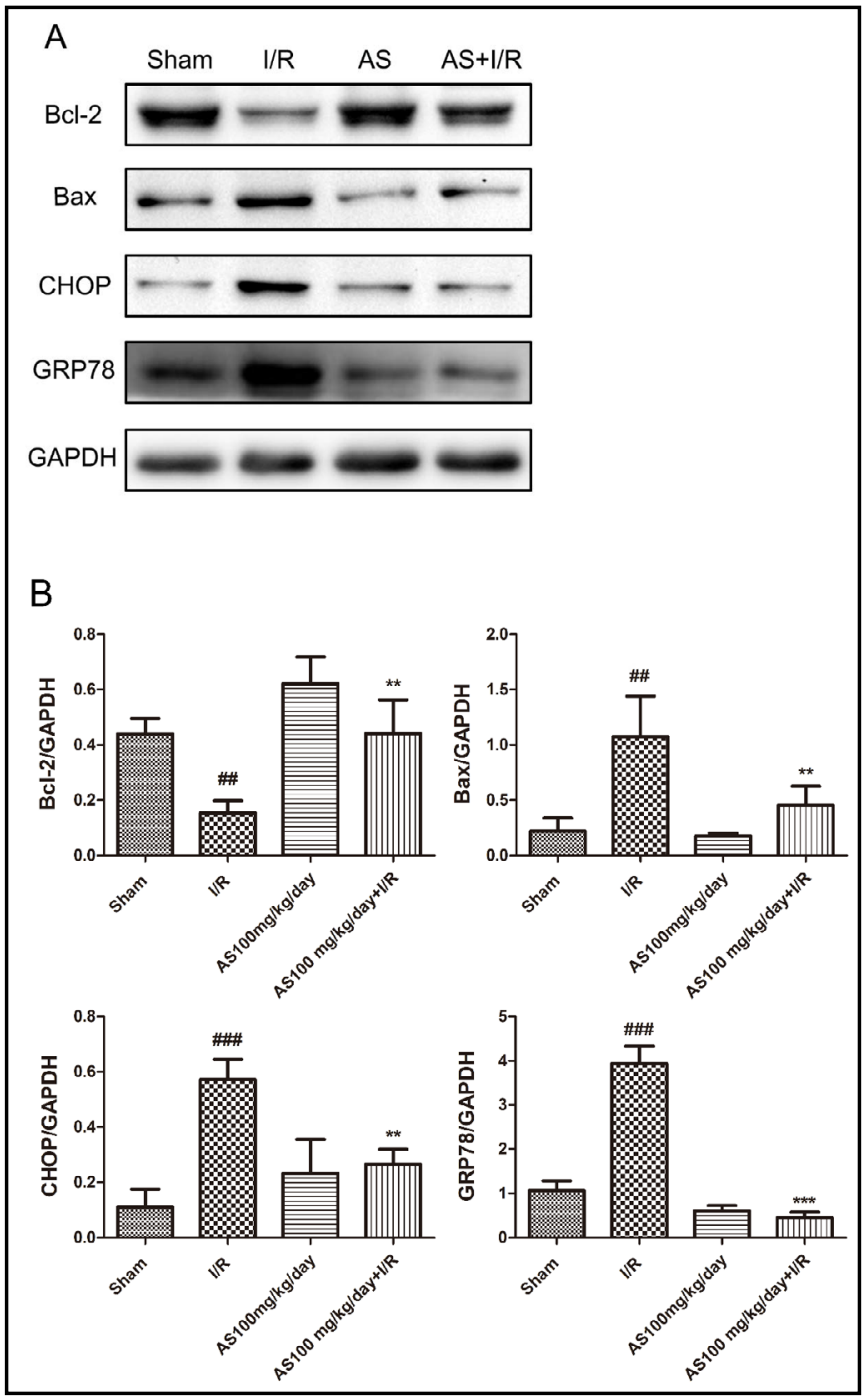




\section{Cellular Physiology Cell Physiol Biochem 2018;50:28-40 \begin{tabular}{ll|l} 
and Biochemistry & $\begin{array}{l}\text { DOI: 10.1159/000493954 } \\
\text { Published onlIne: 3 October } 2018\end{array}$ & $\begin{array}{l}\text { (c) } 2018 \text { The Author(s). Published by S. Karger AG, Basel } \\
\text { www.karger.com/cpb }\end{array}$
\end{tabular}}

Wang et al.: AS Protects MIRI from Calcium Homeostasis Imbalance and ERS

by the effect of anti-oxidation, anti-calcium overload, promotion of energy metabolism and inhibition of apoptosis $[11,24]$. Studies related to AS showed that reperfusion of less than 8 hours merely induced the elevation of LDH, CK and MDA and decline of SOD $[25,26]$, called acute ischemia reperfusion. Early studies have found that with the prolongation of reperfusion time, myocardial injury is gradually aggravating, from pathological changes to the changes of myocardial structure and function [6]. $48 \mathrm{~h}$ reperfusion called as subacute ischemia/reperfusion model, has pathological changes. Therefore, this study chose $30 \mathrm{~min}$ ischemia and $48 \mathrm{~h}$ reperfusion, to explore the cardioprotective effects of AS and its potential mechanisms.

Myocardial infarct size and myocardial enzyme activity are considered as the gold standard for determining the MIRI $[12,27]$. After AS treatment, the infarct size visibly reduced, and the level of LDH and CK also declined in different degrees, which generated consistent results with isolated hearts [28]. The above results directly suggested that AS exhibited protection on MIRI model. According to the result of HE staining, MIR could induce hyperemia, hemorrhage, cardiac reparative inflammation generating, and later hyperplasia of fibrous tissue and myocardial fibrosis [13]. AS treatment group could relieve lesion severity rather than reverse, which was consistent with masson staining in Fig. 4. Besides, LVEDP is related to ventricular volume, diastolic function and ventricular compliance, which can indirectly reflect the left ventricular function [15, 20]. In our study, LVEDP of the AS group tended to return to normal levels compared with the I/R group, which implied that AS could improve left ventricular diastolic function and increase myocardial positive inotropic effect $[15,20]$. In myocardial ischemia, mitochondrial oxidative phosphorylation and ATP production rapidly decreased, when $\mathrm{Ca}^{2+}$ concentration in the mitochondrial matrix increased $[29,30]$. When the heart restored blood oxygen supply, a sufficient number of ROS generated, leading to cell membrane damage and permeability increase [29, 31]. In the study, oxidant and antioxidant indicators were measured. The level of MDA can reflect the degree of lipid peroxidation and SOD as an antioxidase can remove oxidation and peroxidation substance in the body. As showed in Fig. 5, the level of MDA in the IR group increased compared with other groups but the level of SOD decreased, which was consistent with the results of cell and isolated heart. However, AS could significantly reduce the generation of MDA and increase the SOD activity in myocardial tissue, implying that AS could alleviate the oxidative stress induced by MIR and reduce the damage of myocardial cytomembrane [8]. All these results indicated that AS indeed exhibited protection on MIRI model indeed.

Calcium overload is considered as a critical factor of initiating MIRI[32, 33]. Therefore, we detected some ATPase activities associated with calcium to determine their relationship. $\mathrm{Na}^{+}-\mathrm{K}^{+}$-ATPase is an integral membrane protein and an important system of cell energy conversion [34]. Our study showed $\mathrm{Na}^{+}-\mathrm{K}^{+}$-ATPase activity is decreased significantly after MIR. This phenomenon may be due to respiratory depression at the time of myocardial ischemia, hypoxia, and decrease of ATP production. The situation resulted in an increase of intracellular $\mathrm{Na}^{+}$, and then more $\mathrm{Ca}^{2+}$ went into the cell through the $\mathrm{Na}^{+}-\mathrm{Ca}^{2+}$ exchanger, which contributed to calcium overload $[35,36]$. $\mathrm{Ca}^{2+}-\mathrm{Mg}^{2+}$-ATPase was activated when $\mathrm{Ca}^{2+}$ increased to a certain extent, and then $\mathrm{Ca}^{2+}$ pumped out of cells or into the ER, decreasing the intracellular $\mathrm{Ca}^{2+}$. The predominant active transport protein that could regulate intracellular calcium levels $[21,37]$. The results showed that MIR induced the decrease of $\mathrm{Ca}^{2+}-\mathrm{Mg}^{2+}$-ATPase activity noticeable. These findings suggest that probably too much $\mathrm{Ca}^{2+}$ were pumped out of sarcoplasmic reticulum (SR) and did not return to the SR because of calcium pump failure in plasma membrane and SR after MIRI [16]. Our study showed AS administration can visibly elevate the activities of $\mathrm{Na}^{+}-\mathrm{K}^{+}$-ATPase and $\mathrm{Ca}^{2+}-\mathrm{Mg}^{2+}$-ATPase. The above findings showed cardioprotective effects of AS reflected on alleviating the energy metabolism disorder and calcium overload. SERCA is analogous with $\mathrm{Ca}^{2+}-\mathrm{Mg}^{2+}$-ATPase in function but different in structure [38], which is also a key factor of $\mathrm{Ca}^{2+}$ intake in SR. Our data demonstrated MIR induced SERCA activity reduction. It initiated calcium overload and energy metabolic disorders, but AS obviously elevated SERCA activity and improved the condition. Previous studies have shown that SR could control myocardial contraction and relaxation by regulating $\mathrm{Ca}^{2+}$ concentration, and SERCA played a role in the process $[39,40]$. 
These results were also proved in isolated adult rat cardiomyocytes previously by our group. CaN was ubiquitous in cells and was activated by the binding of calcium. Recent studies have shown that CaN played an important role in the apoptosis induced by MIRI [22]. CaN was also reported to be a multifunctional signal enzyme downstream of calcium signaling, and was involved in signal transmission of hypertrophy, proliferation and apoptosis [41]. In our results, AS could suppress the elevation of CaN induced by IR and probably had antiapoptotic effect. However, its apoptosis regulating mechanism and its relationship with other apoptosis signaling pathways were still controversial. From the above results, AS improved the ATPase activity and promoted the restoration of calcium homeostasis after MIRI.

ER is not only involved in protein glycosylation, protein conformational folding, lipid and cholesterol synthesis, but is also important intracellular $\mathrm{Ca}^{2+}$ regulators [42]. ER also manages the release and uptake of $\mathrm{Ca}^{2+}$ mainly through SERCA. Therefore, a close interaction between calcium homeostasis and ERS may exist [43]. When acute myocardial infarction generated myocardial hypoxemia, ATP was rapidly depleted and SERCA activity decreased. The results were $\mathrm{Ca}^{2+}$ in ER was emptied and unfolded protein or misfolding proteins were accumulated in ER, which resulted in ERS[44]. In the early ERS, GRP78, the ERS marker, bound to unfolded proteins in ER and reduced the ER load, restoring homeostasis. If ERS was prolonged and aggravation, CHOP can initiate apoptotic signal pathway $[6,43]$. Our work aimed to study the intervention of AS on ERS by western blot. Our results suggested that the expression of GRP78, CHOP and Bax in the IR group was up-regulation dramatically, whereas Bcl-2 was down-regulation. Up-regulation of CHOP disturbed the balance of proapoptotic and anti-apoptotic gene in the Bcl-2 family and promoted apoptosis [31], which was consistent with our finding. In the AS administration group, the expression level of GRP78, CHOP and Bax decreased, whereas Bcl-2 increased. The above findings indicated that AS probably demonstrated cardioprotective effects by adjusting ERS and its relevant apoptotic pathway. However, our study is not enough to clarify the intrinsic relationship between calcium homeostasis and ERS based on MIRI; therefore, continuous research is essential.

\section{Conclusion}

Our data demonstrated that AS pretreatment exhibited protective effects on MIRI. Our results directly suggested AS could decrease infarct size and improve pathologic progress after MIR. The data also showed that AS exhibited protective effects by alleviating oxidative damage and elevating ATPase activity. Moreover, ERS-relevant signaling pathway played a crucial role on MIRI. In a word, implementing further mechanism research of AS's protection on MIRI is indispensable, beyond that, providing guidance for clinical application, and even broadening the scope of clinical application.

\section{Acknowledgements}

This study was supported by the National Natural Sciences Foundation of China (Grant Nos. 81473380 and 81603333), the Natural Sciences Foundation of Beijing (Grant No. 7152102), the Special Research Project for TCM (Grant No. 201507004), CAMS Innovation Fund for Medical Sciences (CIFMS) (Grant No. 2016-I2M-1-012) and the Program for Innovative Research Team in IMPLAD (Grant No. IT1301).

\section{Disclosure Statement}

No conflict of interests exists. 


\section{Cellular Physiology Cell Physiol Biochem 2018;50:28-40

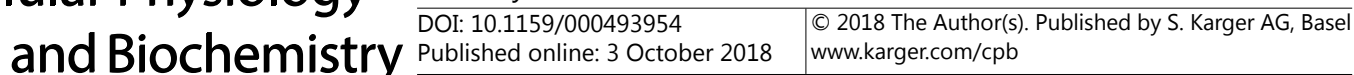

Wang et al.: AS Protects MIRI from Calcium Homeostasis Imbalance and ERS

\section{References}

1 Yu Y, Sun G, Luo Y, Wang M, Chen R, Zhang J, Ai Q, Xing N, Sun X: Cardioprotective effects of notoginsenoside r1 against ischemia/reperfusion injuries by regulating oxidative stress- and endoplasmic reticulum stressrelated signaling pathways. Sci Rep 2016;6:241-250.

2 Zhao J, Tan Y, Jiang T, Lu W: Pharmacological effects of Aralia elata. Jilin Journal of Traditional Chinese Medicine 2016;36:207-210.

-3 Heusch G, Musiolik J, Gedik N, Skyschally A: Mitochondrial stat3 activation and cardioprotection by ischemic postconditioning in pigs with regional myocardial ischemia/reperfusion. Circ Res 2011;109:13021308.

4 Cominacini L, Mozzini C, Garbin U, Pasini A, Stranieri C, Solani E, Vallerio P, Tinelli IA, Fratta Pasini A: Endoplasmic reticulum stress and nrf2 signaling in cardiovascular diseases. Free Radic Biol Med 2015;88:233-242.

5 Mihailidou C, Papavassiliou AG, Kiaris H: A crosstalk between p21 and upr-induced transcription factor c/ ebp homologous protein (chop) linked to type 2 diabetes. Biochimie 2014;99:19-27.

6 Fang S, Li P, Wang C, Xin Y, Lu W, Zhang X, Zuo S, Ma C, Tang C, Nie S, Qi Y: Inhibition of endoplasmic reticulum stress by neuregulin-1 protects against myocardial ischemia/reperfusion injury. Peptides 2017;88:196-207.

7 Wen F, Xu H, Zhang W, Ding T, Sun X: Studies on anti-myocardiac ischemia of aralia elata saponin. World Sci Technol 2005; 7:6-8.

8 Sun G, Xu H, Wen F, Zhang W, Ding T, Sun X: Protective effects of aralosides on cultured myocardial cells subjected to anoxia/reoxygenation injury. Chin Pharmacol Bull 2006;22:1092-1095.

9 Sun G, Wang M, Gao M, Xu H, Sun X: Protective effects of aralosides(as) against h2o2-induced injury in neonatal rat cardiomyocytes. Chin Pharmacol Bull 2013;29:773-777.

10 Luo Y, Dong X, Yu Y, Sun G, Sun X: Total aralosides of aralia elata (miq) seem (tasaes) ameliorate nonalcoholic steatohepatitis by modulating ire $1 \alpha$-mediated jnk and nf- $\mathrm{kb}$ pathways in apoe-/- mice. J Ethnopharmacol 2015;163:241-250.

11 Wang M, Xu X, Xu H, Wen F, Zhang X, Sun H, Yao F, Sun G, Sun X: Effect of the total saponins of aralia elata (miq) seem on cardiac contractile function and intracellular calcium cycling regulation. J Ethnopharmacol 2014;155:240-247.

12 Xiaohui Wang, Tuanzhu Ha, Yuanping Hu, Chen Lu LL, Xia Zhang, Race Kao, John Kalbfleisch, David Williams, Li C: Microrna-214 protects against hypoxia/reoxygenation induced cell damage and myocardial ischemia/reperfusion injury via suppression of pten and bim1 expression. Oncotarget 2016;7:8692686936.

13 Wang C, Liu M, Sun S, Song D, Liu X, Shi D: Panax quinquefolium saponins protect rat myocardium from ischemia /reperfusion injury through attenuating excessive endoplasmic reticulum stress. Chin J Pathophysiol 2013;29:20-27.

14 Chen R, Wang J, Yu Y, Sun G, Sun X: Protective effect of total saponins of aralia elata (miq) seem on lipopolysaccharide-induced cardiac dysfunction via down-regulation of inflammatory signaling in mice. Rsc Advances 2015;5:22560-22569.

15 Li C, Li Y, Zhang D, Wang C, Liu J: Effect of comibination of radix aconiti lateralis and radix ginseng on hemodynamics of acute heart failure rats. Trad Chin Drug Res Pharmacol 2011;22:593-598.

16 Zhang L, Ma J, Liu H: Protective effect of ischemic postconditioning against ischemia reperfusion-induced myocardium oxidative injury in ir rats. Molecules 2012;17:3805-3817.

17 Zhang J, Hou Y, Lu W, Ni X, Lin F, Yu Y, Tang C, Qi Y: Intermedin1-53 protects against myocardial fibrosis by inhibiting endoplasmic reticulum stress and inflammation induced by homocysteine in apolipoprotein e-deficient mice. J Atheroscler Thromb 2016;23:1294-1306.

18 Gao S, Wu R, Zeng Y: Up-regulation of peroxisome proliferator-activated receptor gamma in radiationinduced heart injury in rats. Radiat Environ Biophys 2012;51:53-59.

19 Jia G, Bian Y, Xiao C, Li Z: Adiponectin reduces apoptosis by improving myocardial anti-oxidative activity after myocardial ischemia / reperfusion injury in diabetic rats. Chin Pharmacol Bull 2014;30:623-627.

20 Zhang Y, Huang S, Wang Y, Pan J, Li D, Zheng J, Chen C: Study on protective and haemodynamic effects of danshen tongluo capsule on rats with myocardial infarction. China J Chin Materia Medica 2015;40:528-532.

-21 Chen C, Chen W, Nong Z, Ma Y, Qiu S, Wu G: Cardioprotective effects of combined therapy with hyperbaric oxygen and diltiazem pretreatment on myocardial ischemia-reperfusion injury in rats. Cell Physiol Biochem 2016;38:2015-2029. 


\section{Cellular Physiology Cell Physiol Biochem 2018;50:28-40

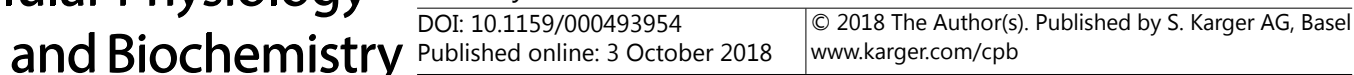

Wang et al.: AS Protects MIRI from Calcium Homeostasis Imbalance and ERS

22 Parra V, Rothermel BA: Calcineurin signaling in the heart: The importance of time and place. J Mol Cell Cardiol 2017;103:121-136.

-23 Stammers AN, Susser SE, Hamm NC, Hlynsky MW, Kimber DE, Kehler DS, Duhamel TA: The regulation of sarco(endo)plasmic reticulum calcium-atpases (serca). Can J Physiol Pharmacol 2015;93:843-854.

-24 Zhang M, Sun G, Xu H, Wang M, Sun X: Effect of aralosides to contraction function and calcium transient of ischemia/reperfusion myocardial cells. China J Chin Materia Medica 2015;40:2403-2407.

25 Lu W, Li M, Wang T: Study of protection mechanism of aralosides on myocardial ischemia/reperfusion injury in rats. China J Traditional Chin Med Pharm 2011;26:554-556.

26 Cui X, Li M, Lu W: Influence of aralosides on cd40l expression in rats with myocardial ischemia and reperfusion. J Beijing Traditional Chin Med 2010;33:323-326.

27 Wu J, Yu J, Xie P, Maimaitili Y, Wang J, Yang L, Ma H, Zhang X, Yang Y, Zheng H: Sevoflurane postconditioning protects the myocardium against ischemia/reperfusion injury via activation of the JAK2-STAT3 pathway. PeerJ 2017;5:3196-3208.

28 Tan Y, Lu W, Zhao J: Study of Effects and Function Mechanism of Aralosides on Mitochondrial Cytochrome C and Membrane Potential of IRI Rat Cardiac Muscle Cells. Western Journal of Traditional Chinese Medicine 2016;29:9-12.

-29 Hong J, Kim K, Kim JH, Park Y: The role of endoplasmic reticulum stress in cardiovascular disease and exercise. Int J Vasc Med 2017;2017:217-225.

-30 Lopez-Crisosto C, Pennanen C, Vasquez-Trincado C, Morales PE, Bravo-Sagua R, Quest AFG, Chiong M, Lavandero S: Sarcoplasmic reticulum-mitochondria communication in cardiovascular pathophysiology. Nat Rev Cardiol 2017;14:342-360.

-31 Szegezdi E, Logue SE, Gorman AM, Samali A: Mediators of endoplasmic reticulum stress-induced apoptosis. Embo Rep 2006;7:880-885.

-32 Xie Y, Gu ZJ, Wu MX, Huang TC, Ou JS, Ni HS, Lin MH, Yuan WL, Wang JF, Chen YX: Disruption of calcium homeostasis by cardiac-specific over-expression of ppar-gamma in mice: A role in ventricular arrhythmia. Life Sci 2016;167:12-21.

33 He X, Bi W, Wang H, Yu X, Zang W: Progress in calcium regulation in myocardial and vascular ischemiareperfusion injury. Acta Physiol Sin 2012;64:321-326.

34 Alison L Müller DF, Dhalla NS: Activation of proteases and changes in Na+-K+-atpase subunits in hearts subjected to ischemia-reperfusion. J Appl Physiol 2012;114:351-360.

35 Singh RB, Hryshko L, Freed D, Dhalla NS: Activation of proteolytic enzymes and depression of the sarcolemmal $\mathrm{Na}+-\mathrm{K}+-$ atpase in ischemia-reperfused heart may be mediated through oxidative stress. Can J Physiol Pharmacol 2012;90:249-260.

-36 Belliard A, Gulati GK, Duan Q Alves R, Brewer S, Madan N, Sottejeau Y, Wang X, Kalisz J, Pierre SV: Ischemia/ reperfusion-induced alterations of enzymatic and signaling functions of the rat cardiac $\mathrm{Na}+\mathrm{K}+$-atpase: Protection by ouabain preconditioning. Physiol Rep 2016;4:12991-13011.

-37 Qiao Z, Ma J, Liu H: Evaluation of the antioxidant potential of salvia miltiorrhiza ethanol extract in a rat model of ischemia-reperfusion injury. Molecules 2011;16:10002-10012.

-38 Dode L, Andersen JP, Raeymaekers L, Missiaen L, Vilsen B, Wuytack F: Functional comparison between secretory pathway $\mathrm{Ca} 2+/ \mathrm{Mn} 2+$-atpase (spca) 1 and sarcoplasmic reticulum ca2+-atpase (serca) 1 isoforms by steady-state and transient kinetic analyses. J Biol Chem 2005;280:39124-39134.

39 Wang C, Liu N, Luan R, Li Y, Wang D, Zou W, Xing Y, Tao L, Cao F, Wang H: Apelin protects sarcoplasmic reticulum function and cardiac performance in ischaemia-reperfusion by attenuating oxidation of sarcoplasmic reticulum ca2+-atpase and ryanodine receptor. Cardiovasc Res 2013;100:114-124.

40 Chernorudskiy AL, Zito E: Regulation of calcium homeostasis by er redox: A close-up of the er/ mitochondria connection. J Mol Biol 2017;429:620-632.

-41 Leshnower BG, Kanemoto S, Matsubara M, Sakamoto H, Hinmon R, Gorman JH, Gorman RC: Cyclosporine preserves mitochondrial morphology after myocardial ischemia/reperfusion independent of calcineurin inhibition. Ann Thorac Surg 2008;86:1286-1292.

42 Guo Y, Li Z, Nan S: The relationship between endoplasmic reticulum stress and myocardial ischemiareperfusion injury. Heilongjiang Med J 2014;37:104-104.

43 Folch-Puy E, Panisello A, Oliva J, Lopez A, Castro Benitez C, Adam R, Rosello-Catafau J: Relevance of endoplasmic reticulum stress cell signaling in liver cold ischemia reperfusion injury. Int J Mol Sci 2016;17:807-818.

44 Li Y, Zhu W, Tao J, Xin P, Liu M, Li J, Wei M: Fasudil protects the heart against ischemia-reperfusion injury by attenuating endoplasmic reticulum stress and modulating serca activity: The differential role for PI3K/Akt and JAK2/STAT3 signaling pathways. Plos One 2012;7:115-126. 